\title{
Understanding How a Culture of Collaboration Develops Among STEM Fac- ulty
}

\section{Dr. Kelly J. Cross, University of Illinois, Urbana-Champaign}

Dr. Cross completed her doctoral program in Engineering Education at Virginia Tech in 2015 and is currently working as a post-doctoral researcher at the University of Illinois at Urbana-Champaign. She is involved with multiple educational research projects with faculty and graduate students at UIUC. Her research interests include diversity and inclusion, teamwork skills, assessment, and identity construction.

\section{Dr. Natasha Aniceto Mamaril, University of Illinois, Urbana-Champaign}

Natasha Mamaril is currently the Coordinator of Undergraduate Research in the College of Engineering at the University of Illinois at Urbana-Champaign. Her research interests include assessment of motivation and how motivation affects student learning. Her education includes a B.S. in Chemical Engineering from the University of the Philippines, and M.S. and Ph.D. degrees in Educational Psychology from the University of Kentucky. She also has nine years of industry experience.

\section{Ms. Nicole Johnson, University of Illinois, Urbana-Champaign}

Nicole received her B.S. in Engineering Physics at the Colorado School of Mines (CSM) in May 2013. She is currently working towards a PhD in Materials Science and Engineering at the University of Illinois at Urbana-Champaign (UIUC) under Professor Angus Rockett and Geoffrey Herman. Her research is a mixture between understanding defect behavior in solar cells and student learning in Materials Science. Outside of research she helps plan the Girls Learning About Materials (GLAM) summer camp for high school girls at UIUC.

\section{Dr. Geoffrey L. Herman, University of Illinois, Urbana-Champaign}

Dr. Geoffrey L. Herman is a visiting assistant professor with the Illinois Foundry for Innovation in Engineering Education at the University of Illinois at Urbana-Champaign and a research assistant professor with the Department of Curriculum \& Instruction. He earned his Ph.D. in Electrical and Computer Engineering from the University of Illinois at Urbana-Champaign as a Mavis Future Faculty Fellow and conducted postdoctoral research with Ruth Streveler in the School of Engineering Education at Purdue University. His research interests include creating systems for sustainable improvement in engineering education, promoting intrinsic motivation in the classroom, conceptual change and development in engineering students, and change in faculty beliefs about teaching and learning. He serves as the webmaster for the ASEE Educational Research and Methods Division. 


\title{
Understanding How a Culture of Collaboration Develops Among STEM Faculty
}

\begin{abstract}
Many stakeholders have called for education reform and particularly regarding the outdated manner in which we generally educate engineers. As a result, understanding dissemination and implementation tactics of research-based instructional strategies (RBIS) has emerged as a critical topic within engineering education research. Additionally funding agencies such as the NSF, have encouraged interdisciplinary projects where STEM faculty work with education experts to apply innovations in teaching. However, how to make these joint ventures successful is less well understood. For example, collaboration in engineering education research typically focuses on student teams or how professional engineering teams learn to work together. Fewer studies articulate how to motivate engineering faculty to interact across engineering disciplines, let alone, with non-engineering faculty such as educational experts. Therefore, the research team sought to understand, how can we develop a culture of collaboration among STEM faculty around the issue of implementing teaching innovation including RBIS's? The specific guiding research question for the current study is how do faculty in STEM describe their experience participating in the Strategic Instructional Innovations Program (SIIP) - a program designed to promote and support the implementation of teaching innovation?
\end{abstract}

This qualitative study employs an exploratory phenomenological approach, using semistructured interviews with 12 STEM faculty across academic ranks. The participants worked on a collaborative team project(s) to implement teaching innovations at a Midwestern large researchintensive, predominantly white institution (PWI). The project durations ranged from one to three years for sustainable implementation of teaching innovations. The semi-structured interviews covered the participant's previous teaching experience prior to joining the SIIP community, a description of their current role in the community including what did and did not work well, and a description of their vision for the community in the future. Consistent with phenomenological research, the interviews were evaluated holistically to allow essential themes of the experience to emerge.

Preliminary results of the phenomenological analysis suggest three emergent themes. First, the participants specified the entry point for implementing instructional innovation. That is to say, the departmental culture was emphasized as a key structural support to ensure the sustainability of the implemented innovation. The second emergent theme articulated by the participants, was the recognition of individual skills and abilities within the SIIP community. Specifically, the expanded peer interaction fostered an environment for complimentary skills to thrive. For example, some of the STEM faculty were more comfortable than others with flipping their classroom, particularly with large service courses with over 100 students, and were able to share best practices or personal success stories. Finally, most participants acknowledge a significant shift in their appreciation for teaching and learning including the scholarship of teaching. In conclusion these combined emergent themes suggest that faculty in STEM require structural and social support such as a community of practice to engage in activities to successfully establish a culture of collaboration when implementing instructional innovation. 


\section{Introduction}

The growing call for engineering education reform led to the investigation of multiple researchbased instructional strategies (RBIS), but the literature suggests a dearth of implementation across engineering educators. Recent engineering education studies indicate a high level of awareness among engineering faculty about highly cited RBIS's, however the implementation and sustained use of the teaching techniques vary significantly based upon the required faculty effort ${ }^{1}$. The two most cited barriers to implementing RBIS's in core electrical engineering courses included 1) concern for requiring too much class time which could potentially undermine their efforts to cover the course content and 2) the cost of preparation time to implement RBIS ${ }^{1}$. As a result, engineering education researchers were urged to provide details of implementation and the underlying educational research principles in addition to evidence of efficacy when attempting to convince STEM faculty to apply RBIS's in their classroom ${ }^{2}$. Potential adopters should seek out the background information to make informed decisions about how to properly implement the RBIS within the course and achieve the desired student outcomes ${ }^{2}$. Furthermore, the obstacles impeding wide spread integration into the engineering education seem to occur at later stages of adoption beyond awareness and implementation such as a sustained effort to continue the use of the RBIS or discontinue ${ }^{1,3}$. Therefore, it is important to understand how to sustain the implementation of RBIS's and the research-to-practice gap in the context of engineering curriculum.

Related to the general lack of sustained implementation of RBIS's in STEM is the research-topractice gap in engineering education. Although, European education researchers covered the research-to-practice gap ${ }^{4}$, the topic has been less covered in the United States engineering education literature. Few engineering education studies explicitly connect research based instructional practices directly to practitioners with the exception of cooperative learning literature ${ }^{5,6}$ and the 141 conference papers published with the American Society of Engineering Education since 2010. Recently, engineering educators at the University of Michigan developed an institutional change model or plan to bridge the research-to-practice gap ${ }^{7}$. The authors emphasized minimizing faculty effort by developing easy to implement active learning techniques to allow faculty to take incremental steps throughout the initial implementation and maintained adoption ${ }^{7}$. Furthermore, building a learning community structurally supported faculty in STEM sharing information, resources, and responsibility to collectively develop solutions ${ }^{7}$. Although Finelli et al. (2014) connected the study results to the local college of engineering culture, we argue that building a community among STEM faculty is a necessary step in implementing RBIS's and could lead to the development of instructional innovation.

The Strategic Instructional Innovations Program (SIIP) was created in the College of Engineering at the University of Illinois at Urbana-Champaign to create a community of faculty who would develop new instructional innovations and sustainably implement RBISs. Supplemented by NSF funding to extend to other STEM departments, faculty broker these changes by developing collaborative, joint ownership of their courses. It was believed that developing this collaborative joint ownership, akin to a Community of Practice (COP) ${ }^{8}$, would provide a means for faculty to share common interests and passions for improving their courses and then subsequently sharing knowledge and best practices to accelerate learning and change ${ }^{9}$. These smaller, course-centric communities (henceforth, called innovation COPs to reflect the 
language used among the faculty in the program) were organized into a larger SIIP-wide community that sought to facilitate knowledge sharing across these innovation COPs. While each innovation COP was composed of three to nine faculty members, SIIP as a whole has engaged over 200 faculty members from 15 departments, creating a fairly rich and complex organizational structure. The goal of this research study was to understand and characterize the experience of faculty, identified as leaders or change agents, during the development of a collaborative community within the SIIP program designed to implement instructional innovation throughout the college of engineering.

Studying organizational change such as the implementation of instructional strategies through a collaborative community can be characterized by a phenomenological study of the organization. Recent research suggest that phenomenology is an effective strategy of inquiry to investigate organizational identity or institutional change work ${ }^{10}$. According to Gill (2014), researchers could capitalize on the epistemological underpinnings of phenomenology to uncover nuances of the experiences of the organization members. In other words, the individual STEM faculty experience can illuminate the institutional and social reality that supports the shared passion for organizational change. Furthermore, social science researchers suggest that the concepts of phenomenology are a rich source of explanatory power to understand organizations and institutional characteristic ${ }^{10,11}$. As a result, our decision to apply a phenomenological approach is consistent with previous research that aimed to study organization or institution characteristics. Therefore, the phenomenology approach is a key component of the study design and was a critical methodological decision to develop answers to the overall research question of how faculty in STEM develop a collaborative change community with the objective to improve the education of undergraduate engineering students by adopting RBIS or developing instructional innovations.

\section{Methods}

This qualitative study employed an exploratory phenomenologically informed approach to understanding collaboration amongst STEM faculty [with the support of university administration]. The work consisted of semi-structured interviews with 12 STEM faculty across academic ranks who had been or are currently working on a variety of collaborative team projects to implement teaching innovations at a large Midwestern research-intensive institution.

\section{Participants}

A purposeful sampling approach was used to study the participants who were identified as leaders of the teaching innovation program hosted by the college of engineering $(\mathrm{N}=12)$. The participants were initially contacted by email and then interviewed in person. The participants came from a variety of departments and represented a mixture of diverse demographic factors including gender, professional experience, academic standing (academic professionals vs. tenure track professors vs. administrators) and teaching levels (large introductory courses vs. smaller upper division courses). The broad range of previous research and teaching experience increased the chances of reaching saturation. All the participants played distinct but overlapping roles within the leadership team for the project. While most participants were engineering faculty, one identified leader in the community came from the Geology Department. The final pseudonym list of participants is in Table 1. Please note that due to the small number of participants and close 
professional relationships, the listed pseudonym does not necessarily reflect the gender of the participant.

Table 1: Participant Pseudonym List

\begin{tabular}{|c|c|c|}
\hline Jack & Cynthia & Ashley \\
\hline DeWhite & Peyton & Sarah \\
\hline James & Jamie & Ricky \\
\hline Marge & Taylor & Tim \\
\hline
\end{tabular}

\section{Data Collection}

Qualitative data was gathered through semi-structured in-person interviews. The goal of the interviews was to elicit the participant's self-perceived experiences within the collaborative community to understand similarities across the group. Each participant was interviewed by one of three experienced interviewers using a collaboratively constructed interview protocol. Composed of open-ended questions, the protocol allowed participants to describe her/his experiences in her/his own way. The interviews included items such as 1) background information about the participants, 2) participants' self-reported role and relationships with others in the program and 3) a description of what did and did not work well within the overall program in addition to his/her future vision of the program. Each interview was audio recorded and later transcribed verbatim. The interviews typically lasted between 45-60 minutes and the research team scrubbed identifying information to maintain anonymity of the participants.

\section{Data Analysis}

Data analysis consisted of 1) using open coding to construct a codebook to capture the variety and nuances of the participants' experiences based on a random selection of participants ${ }^{12}$ and 2) then axial coding, using the codebook to holistically evaluate each participant's interview. Essential themes representative of the participants' experiences as part of the group emerged using a thematic analysis ${ }^{13}$. First, three of the twelve interviews were randomly selected for open coding. Each member of the three-person research team then coded the interviews independently using the word processing software, MaxQDA, where sections of texts were labelled with codes. The research team created analytical memos during the coding process to support later meaning making or to consult during analysis. An example of open coding is the text from Sarah "So we decided to have this [program] Celebration of Teaching where we would invite the [junior faculty teaching program name], the department heads to come and like get their certificates and we also decided to have the [program] teams come and show - have a poster session. So, it worked- it was really good. It worked out really well. There was a really nice energy to it and people showed up. The dean was there and it was a very positive thing" being coded as "Administrator Buy-in." After each member coded the three interviews, similar codes were grouped and renamed until fifteen unique codes materialized. Codes that captured nuances of one or two people but did not reflect a general trend across the group were either removed or combined with other, similar codes. The final list of codes became the basis of the codebook to be used in the rest of the analysis once the group members mutually agreed upon the scope and level of detail of each code. Following the process detailed in MacQueen et al. ${ }^{12}$, each 
code in the codebook was given a brief and full definition as well as guidelines for when and when not to use the code and example texts where the code was used.

Next, a critical part of data collection process when conducting a phenomenological study is managing bias. Bias in the analysis was minimized using intercoder agreement as a way to account for individual bias during coding. After an initial codebook was created, each member of the research team independently coded randomly selected interviews. The results of the independent coding was evaluated using the MaxQDA analysis function to measure intercoder agreement. The intercoder agreement calculation generated a value between 0 and $100 \%$ which compared the coded segments between researchers based on both the assigned code name and the text coverage. The research team discussed disagreements until a consensus was reached. After three rounds of independent coding and comparison, intercoder agreement on text segmentation was consistently above $80 \%$ across all three group members. Once achieving an acceptable level of intercoder agreement, all twelve interviews were independently recoded using the codebook. The final overall coding agreement among all three members of the research team reached $81 \%$ and meets the recommended level of intercoder agreement ${ }^{14,15}$. After re-coding all the interviews with the operationalized codebook definitions, the coded segments from the interview transcripts were further evaluated to identify emergent themes. The primary emergent themes are discussed in detail in the results section below, however the full set of emergent themes and complete codebook will be part of the upcoming publication.

\section{Results and Discussion}

The discussion is organized by the three interrelated themes: 1) how the participants became involved with the program, 2) their team dynamics including interactions with the innovation COPs and the program leadership, and 3) the shift in their appreciation for teaching and learning expressed through their current perspective on teaching. Also, we briefly describe the participant's future vision for the overall program. Below is an abbreviated version of the codebook (Table 2) that characterizes the three themes including a condensed definition and example quotes of participant's responses to interview questions.

\section{Table 2. Abbreviated Codebook}

\begin{tabular}{|l|l|l|}
\hline Code Name & Brief Definition & Example Quote \\
\hline Entry and First Contact & $\begin{array}{l}\text { Awareness of program and } \\
\text { circumstances of first joining the } \\
\text { program }\end{array}$ & $\begin{array}{l}\text { it was [NAME] saying, "Hey, you } \\
\text { should pay attention to this," I'm } \\
\text { like, "Absolutely, yeah," I started } \\
\text { out more as a participant and } \\
\text { switched quickly to the leadership" }\end{array}$ \\
\hline Team Dynamics & $\begin{array}{l}\text { Interpersonal communication and } \\
\text { interactions that occur within the } \\
\text { leadership team that support the } \\
\text { idea of shared leadership }\end{array}$ & $\begin{array}{l}\text { "We come together and we } \\
\text { discuss things, and here's the } \\
\text { direction, and it's been a } \\
\text { wonderful kind of, flat } \\
\text { organization." }\end{array}$ \\
\hline Current Perspective on Teaching & $\begin{array}{l}\text { Views (static and/or dynamic) on } \\
\text { teaching, teaching philosophy and }\end{array}$ & $\begin{array}{l}\text { "I think communities of practice } \\
\text { are very effective in sustaining }\end{array}$ \\
\hline
\end{tabular}


Next, each theme is discussed in detail with multiple participant's statements that best capture the essence and core meaning of how the members of SIIP describe their experience. Finally, we conclude with a brief synthesis of interrelated ideas between the emergent themes generated by the study and our current understanding of how communities of practice exist among faculty in STEM, committed to improving engineering education.

\section{Entry and First Contact}

Participants described their experiences that led to their awareness and knowledge of the program goals and their initial introduction to the leadership team. Most of them were approached by administrative personnel (e.g., dean, director) for ideas for educational initiatives. For example, Ashley mentioned that "dean [name] wanted to put some college money into strategic research initiatives and to put some money into teaching...we oughta be putting a million dollars a year into making instruction better to our students and that was kind of it. It's like my ball to, the ball was in my court to figure out what to do." Other participants were teaching courses and interested in ways to improve their teaching. Previous teaching experiences led them to engage in discussions on instructional strategies. In particular, James talked about being very unsatisfied with the way he has been teaching his classes based on student performance and then he started having conversations with two other professors to talk about ideas that they were developing, specifically what they were doing in their classes and having their own instructional innovation team. These conversations turned into weekly meetings that focused on teaching "especially for the younger, assistant professors, a lot of it was just "How do I deal with this problem?" as James put it. Some learned about the program on their own through college/university announcements, meetings, or through their colleagues. Tim recalled initially talking to his colleagues about a class that his department faculty was unhappy with and they all thought they could do something about that class, "And so, we went along to... I guess there was a meeting... about this new program that I think [name] was running." Peyton had met with a physicist with training in physics education research (PER) and said "we both saw the need for research and substantive change in instruction and so we sort of proposed to our colleges, engineering and education, that we institute more of an on-going program and sort of the line of argument was if this is an R-1 university where we are doing big time research on stuff, the university itself should be conducting research on its fundamental mission, so we should be conducting research as a university on teaching and education."

During the interviews, participants also talked about a general desire or willingness to join, collaborate with and/or act in a leadership role. They spoke of personal or professional reasons for being part of the program. Taylor's motivation to be part of the program stemmed from the interest to connect with others to collaborate on teaching.

I was quite interested in the [program] process because we've been trying things out in our own numeric sequence here and connecting with all of the other groups in the college was going to be important for that, there were things going on in similar areas that we could benefit from in my own area so that was a big driving point. The other piece was the ability to collaborate on teaching and how we can improve our own courses that was a big impact. 
Jamie's response reflected a desire to learn and improve the curriculum.

So it was a good opportunity, you know, and my motivation with doing it for [course] was actually for me to learn...I figured it would be a good opportunity for me to learn how to do it, and that I would be able to then replicate that in [course], ... But the motivation for doing this [program] thing was the... It was largely about the computation. Computational Modules was an idea that we had...really saying: computation is a key part of material science and material science education.

Overall, personal interest and/or professional networking was a source of motivation to join the program. The participants saw how the program/project goals aligned with their goals (i.e., improve teaching and learning) and recognized benefits from participating in the program.

\section{Team Dynamics}

Although the study participants were identified as leaders of the instructional innovation program, they gave an account of both their experiences as part of the leadership team and their role as part of innovation COPs. Their interview responses included descriptions of oral and written interactions 1) amongst members of the leadership team that adopted a shared sense of leadership, and 2) between members of the leadership team managing or supporting the innovation COPs. Leadership dynamics focused on program management, decision-making, and the collective responsibility of the program. As Cynthia pointed out, decisions were made from who gets funding to who needs to be involved in the program.

Well the decision making process was decided when we wrote the grant because you have to say who you're going to fund and so that really was the case of talking to every unit and having multiple discussions with every unit, we were careful, we talked to every unit and we said, you know "who's involved in undergraduate education, who's somebody who's going to be committed and interested" and then you know you get a small number of faculty.

Jack also described the leadership, "We're a very distributed kind of leadership...which is the way, when you heard this thing about empowerment, in there. Engineering is extremely hierarchical here. On the other hand, the [program] team has always been very distributed. So, it's not like any of us come in with a 'this is the way were gonna do things.' And we all respect everybody's knowledge."

In considering who should be involved in the program, the participants recalled bringing in people with a particular skillset or knowledge base. Sample responses such as these show that they recognized their peers' contributions to the project goal: “... why we decided on various people is like [name] came in late because we wanted to have a very strong evaluation portion and we decided that one great way to do that was to bring [name] into the group and not just have her as an external person" and "without [name] the proposal would never have flown and it would not have had the flavor...he wrote $75 \%$ of the proposal, which was the social aspect of the communities of practice... he learned all that literature. He put it together."

Interactions between the leadership team and the innovation COPs were also explicitly described by participants. For example, Peyton articulated interacting with innovation COPs during the 
proposal review process,

.... a pre-proposal, or white paper, is submitted and the [program] staff and sort of broader [program] review community look at them, decide which ones have merits based on the criteria and select a subgroup for full proposal writing and in the full proposal writing phase, it's a little unique in this way, some member of the staff is assigned to each project and they're required, or strongly encouraged, to meet weekly with their representative from the team. Meeting with them and sort of guiding them through the proposal process, hoping to ensure high-quality final proposal, making sure it includes all things to be addressed by the RFP and that sort of addresses any obstacles.

Similarly, Jack discussed the mid-year review, We had a midyear evaluation, in January where we brought people in. And the PI's had to write a little report. And we kind of, we brought them into a panel like meeting. Where the three or so of us and we ask questions... so all the PI's came and they talked to us. So there was kind of, I mean, you heard in that meeting, that everything is a success. So everybody tries to put their best foot forward, but in this team. At the other side of the table of the [program] people was the person who was embedded into that team, and they knew what was going on. So they would ask very poignant questions, you know. Whenever, some teams are making great progress. And you'd ask questions and they answer them fine. Other times you'd say, but have you met? And they would say, well we met once, you're supposed to be meeting weekly...So, you know, they would have to explain why things were not happening...

From the participants' responses, teamwork was key in getting things done. At different points of the program, they shared the leadership task of making decisions and leveraging members' strengths. The leadership team also provided guidance and structure (e.g., goals, meetings, expectations, mid- year review) to the innovation COPs to help them succeed and fostered an expectation of cooperation among the members of the SIIP community.

\section{Shift in Appreciation of Teaching and Learning}

Another theme that emerged from the data analysis is that most participants acknowledged a shift in their appreciation for teaching and learning as a result of working on an instructional innovation project. Some participants, like Cynthia, expressed how much they valued the application of the scholarship to teaching, particularly using engineering education literature,

Well the other thing I think is really important and that is completely neglected is that we should use the pedagogy literature and that's been very powerful to me and it's been powerful both as instruction about teaching is what we should do, what the limitations of our knowledge about what works is, how modest we should be in our expectations, but also you know you have evidence of what works... So many professors are unaware that there is literature on learning either, I didn't until about five years ago, I didn't know anything, it might have been even four years ago, actually now that I think about it. I never read the literature at all and now I'm not saying I'm expert, you don't have to be an expert, but you do need to be at least conversant with some of the ideas. And this is all stuff that is based in careful research by careful researchers and people dismiss it. And then if we are going to make changes though, we should use literature. 
Jack acknowledged, "what has changed was my perspective on how faculty, whose main focus is really research in their own fields can come to embrace, that you can teach better and that you can learn, that there's literature out there...that you can change systematically, and you can, you know, the old "teach like you do research."

Participants also described their current perspectives on teaching and what they have learned after being involved in the program. For example, Taylor thought about revising the course to improve student outcomes.

I think I've learned three things after being involved with the [program] over the last, [program] projects over the last year, one is that there, the importance of focusing on student outcomes when thinking about revisions to the course, that's difficult to do, but it's also an easier thing too, it can be a concrete thing to put your eyes on. And I think that's missed, I've missed that for myself in revising a course. Usually, I focus, I mean there's so much effort just to get content going in a course and it's helped to re-think, you know, what some of those objectives are so that's really helpful to see how other groups approach that. I think another piece that I've learned is just the vast number of things that you can do in a course to improve those student outcomes. And not all of them need to be immediately in front of the classroom and not all of them need to take a lot of time. And to me that was very enlightening and it's something that I think our department has benefitted from just being more closely connected to, to the resources of the [program] teams.

This perspective was also echoed by Tim, "So now I think I've been putting a lot more effort into, you know, a lot more interest in teaching and how to do it well and things like that, so, there was a big change." Another perspective on teaching involved James' realization that teaching is "much more a group activity now...I also see it as something to talk with other faculty members about."

The idea of teamwork and collaboration was frequently mentioned by the participants during the interviews. Specific references to coordinated or cooperative efforts by a group of people acting as a team were made. For example, Jack talked about how sharing information and talking with peers led to a proposal.

So, there was such a meeting organized. And, uhh, you know, [name] was there and [name] and me. And [name], was also the co-PI, [name] who is a co-PI, and maybe five or six others. And we started discussing things, and uhh, we decided that we were going to go after, a proposal at that meeting. And then [name], suggested that I be the PI, and we, the four of us, [name], [name], [name], and myself, where the core people who wrote it.

James detailed the need for a team to make the program work. "So, we knew that in order to make this work, it was going to require a team; it was going to require multiple people who work with computation to help, because $I$ don't want to be the only person. I don't want to be the one person making all of this stuff; it's way too much...And the other thing about having the team was, it makes it so much easier for faculty that are not in computation to do it. It makes them feel but I would imagine that if I were in their shoes I would feel a lot more comfortable 
about doing something like this knowing that there is a group of people that will help me along the way, that there's support there in terms of other faculty, and then, from us, from the other side, it makes it easier for us to make sure that these things continue and keep going. I don't know, you know I had very few conversations about teaching with anybody, really, up until about a little less than two years ago."[When he joined the COP]

In summary, participants' statements seemed to promote working together and learning from others in the community to improve teaching practices. They realized that collaborating with their peers and colleagues can facilitate the efficient implementation of instructional strategies. Moreover, knowing that there are resources (e.g., literature) and support (e.g., social) available eased their concerns and gave them confidence to attempt modifying their instructional strategies.

\section{Future Vision}

The final but important emergent theme highlighted the leadership team's vision for the overall program going forward in the future. As leaders of the program, participants were intentionally asked to articulate their future vision for the program. Ashley simply said, "...to continue and to thrive, and to keep fostering improvements in teaching in the college," whereas Cynthia "would like to see this idea that we can have these communities of practice sustained." On the other hand, Taylor would like "to see them reach more groups of people that may not have the intention of improving their curriculum." These responses give a snapshot of the scope of the participants' future vision for the program. In essence, they would like to see 1) program longevity, 2) other departments and/or colleges in the program, and 3) and more teams involved in improving teaching. Therefore, the participants saw their COP for instructional innovation to be critical to the success of the college with the potential for sustainability.

The emergent themes are connected in specific ways. First, the initial entrance into to the community was based upon personal motivation and values to improve undergraduate education. With the shared concern to improve undergraduate education, the core group laid the foundation to create an innovation COP with an initial discussion. The initial leadership combined and leveraged their individual talents into a compelling collective effort summarized in a proposal. Once the community was established, the core leadership carefully considered which faculty members to bring into the community dialogue. As the community grew, the sharing of information and resources expanded. In particular, the incoming faculty members enjoyed being able to talk through ideas for instructional innovation and get peer feedback including tacit knowledge such as best practices during implementation. As the community grew, the potential concerns that undermine the community became salient and easier to mitigate. The community with shared responsibility, decided to proactively approach process improvement through assessment. As a result, the community interactions directly impacted the performance of the practitioners within the group. The iterative nature of the steps to create, build, and grow the community positioned the COP to achieve their shared goal of improving engineering education at the undergraduate level at their institution. Additionally, the interactions and collaborations among the participants enhanced the creativity of the group as several members have developed viable options for the future direction of the community and the program as a whole.

Therefore, establishing the COP was the essential organizational support to allow this group to 
successfully engage in instructional innovation to address their shared motivation to improve the undergraduate curriculum.

\section{Conclusions}

In conclusion, the participants described their experience in the COP to improve their undergraduate curriculum as collaborative and cooperative with a shared leadership. The participants generally joined the community based upon their own personal concern to improve engineering education. Importantly, the community structure provided the community with an organized information sharing resource. Specifically, they like being able to talk about their teaching with other faculty or ideas they had about adopting a research-based instructional strategy. The regular interactions supported this sharing and generated creativity that would be difficult to replicate by a single faculty member. Also, the sharing of information shifted the perspective of several members of the community about the scholarship of teaching. Eventually, the collective group agreed that process improvement was important which led to a clarification of the assessment criteria and evaluation process. Therefore, the COP provided organizational structure to support STEM faculty implementing instructional innovation and adopting RBIS's without being overburdened. Furthermore, the emergent themes are interrelated as each theme was the stepping stone for the next community building step that established a well-functioning and sustainable community of faculty and staff in STEM engaged in implementing instructional innovation. Notably the steps in building the community are consistent with team building literature commonly found in engineering education.

\section{Study Limitations}

As with any study, study limitations define the scope of the study design and interpretation of the results. One limitation is the study was conducted at a single institution and future work should consider specific institutional context when translating the efforts in study to a different institution. Also, not all faculty that originally created the community was included in this study, some members are no longer associated with the program. The members that left the community may offer perspective that are not present in the current data set and resultant analysis. Another limitation is that the students affected by the changes in the curriculum are not included in the current study. Future work can include student data to corroborate the success of the implemented instructional changes. A final limitation is that the participants were interviewed only once for this study. It is possible that some details or a critical incidence may not be present in the data set, as a result of limited recall by the participants. Although these limitations are important they do not diminish the significance of the overall findings in the study of the role of COP to support improvement in engineering education, rather they indicate areas for future work.

\section{Acknowledgments}

This work was supported by the National Science Foundation under grant DUE-1347722. The opinions, findings, and conclusions do not necessarily reflect the views of the National Science Foundation or the author's institution.

\section{References}


1 Froyd, J. E., Borrego, M., Cutler, S., Henderson, C. \& Prince, M. J. Estimates of use of researchbased instructional strategies in core electrical or computer engineering courses. IEEE Transactions on Education 56, 393-399, doi:10.1109/TE.2013.2244602 (2013).

2 Borrego, M., Cutler, S., Prince, M., Henderson, C. \& Froyd, J. E. Fidelity of Implementation of Research-Based Instructional Strategies (RBIS) in Engineering Science Courses. Journal of Engineering Education 102, 394-425, doi:10.1002/jee.20020 (2013).

3 Henderson, C., Dancy, M. \& Niewiadomska-Bugaj, M. Use of research-based instructional strategies in introductory physics: Where do faculty leave the innovation-decision process? Physical Review Special Topics-Physics Education Research 8, 020104 (2012).

$4 \quad$ Vanderlinde, R. \& van Braak, J. The gap between educational research and practice: Views of teachers, school leaders, intermediaries and researchers. British Educational Research Journal 36, 299-316, doi:10.1080/01411920902919257 (2010).

5 Johnson, D. W., Johnson, R. T. \& Smith, K. Cooperative learning returns to college what evidence is there that it works? Change: the magazine of higher learning 30, 26-35 (1998).

6 Johnson, D. W., Johnson, R. T. \& Smith, K. The state of cooperative learning in postsecondary and professional settings. Educational Psychology Review 19, 15-29 (2007).

7 Finelli, C. J., Daly, S. R. \& Richardson, K. M. Bridging the Research-to-Practice Gap: Designing an Institutional Change Plan Using Local Evidence. Journal of Engineering Education 103, 331-361 (2014).

8 Wenger, E. Communities of practice: Learning, meaning, and identity. (Cambridge university press, 1999).

9 Wenger, E. Communities of Practice and Social Learning Systems. Vol. 7 (2000).

10 Gill, M. J. The possibilities of phenomenology for organizational research. Organizational Research Methods, 1094428113518348 (2014).

11 Kupers, W. Embodied "inter-learning"-an integral phenomenology of learning in and by organizations. The Learning Organization 15, 388-408 (2008).

12 MacQueen, K. M., McLellan, E., Kay, K. \& Milstein, B. Codebook development for team-based qualitative analysis. Cultural Anthropology Methods 10, 31-36 (1998).

13 Braun, V., \& Clarke, V. Using thematic analysis in psychology. Qualitative Research in Psychology, 3, 77-101. (2006).

14 Creswell, J. W. Research Design: Qualitative, Quantitative, and Mixed Methods Approaches. (Sage Publications, Inc., 2009).

15 Miles, M. B. \& Huberman, A. M. Qualitative data analysis: An expanded sourcebook. (Sage, 1994). 
